\title{
Erratum to: Evaluation of S-1 as third- or further-line chemotherapy in advanced non-small-cell lung cancer
}

\author{
Akira Ono - Tateaki Naito - Haruyasu Murakami - Toshiaki Takahashi • Yukiko Nakamura • \\ Asuka Tsuya $\cdot$ Kyoichi Kaira $\cdot$ Satoshi Igawa - Takehito Shukuya $\cdot$ Akihiro Tamiya • \\ Rieko Kaira • Masahiro Endo • Nobuyuki Yamamoto
}

Published online: 8 April 2010

(C) Japan Society of Clinical Oncology 2010

Erratum to: Int J Clin Oncol

DOI 10.1007/s10147-010-0034-0

The correct name of the ninth author should be given as

Takehito Shukuya, not Takehiro Shukuya.

The online version of the original article can be found under doi:10.1007/s10147-010-0034-0.

A. Ono $\cdot$ T. Naito $\cdot$ H. Murakami $\cdot$ T. Takahashi .

Y. Nakamura $\cdot$ A. Tsuya $\cdot$ K. Kaira $\cdot$ S. Igawa $\cdot$

T. Shukuya $\cdot$ A. Tamiya $\cdot$ R. Kaira $\cdot$ N. Yamamoto $(\bowtie)$

Division of Thoracic Oncology, Shizuoka Cancer Center,

1007 Shimonagakubo, Nagaizumi-cho, Sunto-gun,

Shizuoka 411-8777, Japan

e-mail: n.yamamoto@scchr.jp

M. Endo

Division of Diagnostic Radiology, Shizuoka Cancer Center,

1007 Nagaizumi-cho, Shizuoka 411-8777, Japan 